\title{
Visualization of noise current propagation in a power module with scanning time- synchronized near magnetic field measurement
}

\author{
Takaaki Ibuchia), Eisuke Masuda, and Tsuyoshi Funaki \\ Osaka University, Division of Electrical, Electronic, and Information Engineering, \\ Graduate School of Engineering, 2-1 Yamadaoka, Suita, Osaka 565-0871, Japan \\ a)ibuchi@eei.eng.osaka-u.ac.jp
}

\begin{abstract}
This study investigates noise current propagation in a power module accompanied with its switching operation. The noise current can be estimated from the measured near magnetic field. The proposed measurement system is developed to identify the time-dependent near magnetic field distribution in a power module. The system scans near magnetic field spatially and acquires its time-synchronized frequency spectrum. Propagation of the noise current in the power module is visualized as a time series of the noise current spectrum distribution in the power module. The developed system can help realize electromagnetic compatibility (EMC) design of a power module.
\end{abstract}

Keywords: current density, electromagnetic interference (EMI), near magnetic field intensity, power module design

Classification: Power devices and circuits

\section{References}

[1] C. N. M. Ho, et al.: "A comparative performance study of an interleaved boost converter using commercial Si and SiC diodes for PV applications," IEEE Trans. Power Electron. 28 (2013) 289 (DOI: 10.1109/TPEL.2012.2197830).

[2] D. Han, et al.: "Comprehensive efficiency, weight, and volume comparison of SiC- and Si-based bidirectional DC-DC converters for hybrid electric vehicles," IEEE Trans. Veh. Technol. 63 (2014) 3001 (DOI: 10.1109/TVT. 2014.2323193).

[3] Z. Zhang, et al.: "Evaluation of switching performance of SiC devices in PWM inverter-fed induction motor drives," IEEE Trans. Power Electron. 30 (2015) 5701 (DOI: 10.1109/TPEL.2014.2375827).

[4] N. Oswald, et al.: "An experimental investigation of the tradeoff between switching losses and EMI generation with hard-switched All-Si Si-SiC and All-SiC device combinations," IEEE Trans. Power Electron. 29 (2014) 2393 (DOI: 10.1109/TPEL.2013.2278919).

[5] Z. Fang, et al.: "Impact of application of SiC devices in motor drive on EMI," 2017 IEEE Applied Power Electronics Conference and Exposition (APEC) 29 (2017) 652 (DOI: 10.1109/APEC.2017.7930763). 
[6] D. P. Sadik, et al.: "Analysis of parasitic elements of $\mathrm{SiC}$ power modules with special emphasis on reliability issues," J. Emerg. Sel. Top. Power Electron. 4 (2016) 988 (DOI: 10.1109/JESTPE.2016.2585666).

[7] G. Regnat, et al.: "Optimized power modules for silicon carbide mosfet," IEEE Trans. Ind. Appl. 54 (2018) 1634 (DOI: 10.1109/TIA.2017.2784802).

[8] T. Ibuchi, et al.: "Visualization of noise current distribution in an $\mathrm{SiC}$ power module using near-field magnetic scanning," IEICE Commun. Express 6 (2017) 584 (DOI: 10.1587/comex.2017XBL0106).

[9] T. Ibuchi, et al:: "A study on packaging design of $\mathrm{SiC}$ power module using near-field magnetic scanning techniques," 2017 IEEE International Workshop on Integrated Power Packaging (IWIPP) (2017) 1 (DOI: 10.1109/IWIPP.2017. 7936770).

[10] J. B. Allen: "Short term spectral analysis, synthesis, and modification by discrete Fourier transform," IEEE Trans. Acoust. Speech Signal Process. 25 (1977) 235 (DOI: 10.1109/TASSP.1977.1162950).

\section{Introduction}

Recent development of silicon carbide $(\mathrm{SiC})$ power devices enables fast switching operation of high voltage and large current $[1,2,3]$. Fast transitions of voltage and current induce electromagnetic interference (EMI) noise by interacting with circuit parasitic components $[4,5]$. This problem must be assessed more carefully when designing $\mathrm{SiC}$ power modules $[6,7]$. Identification of the EMI noise source and clarification of noise propagation in a power electronics circuit is important for reducing EMI noise levels. Our previous study $[8,9]$ evaluated the effect of an embedded DC-link snubber capacitor in the tested power module on suppressing the voltage overshoot and high-frequency noise current via the near magnetic field intensity measurement. A power electronics circuit generally operates with a constant switching frequency and phase in a periodic steady state. This report outlines the developed measurement system and visualizes noise current propagation in a power module associated with its periodic steady-state switching operation based on the scanned time-synchronized near magnetic field measurement. This study demonstrates the visualization of noise current propagation in a power module for a single-phase DC-DC full-bridge inverter with inductive load.

\section{Time-synchronized near magnetic field scanning system for evaluating dynamic current distribution}

Fig. 1(a) shows the conventional configuration of a 2D near magnetic field scanning system. A magnetic probe is connected to a spectrum analyzer in a measurement system. A personal computer (PC) controls the position of a magnetic probe over a pre-selected scan surface, which is in the near field of the device under test (DUT), and records the measured data. The magnetic field intensity is measured in two orthogonal directions $\left(H_{x}, H_{y}\right)$ at each measurement point; then, the magnetic field amplitude $H_{x y}$ is calculated. The current density distribution can be visualized based on the scanned $H_{x y}$. The swept-tuned spectrum analyzer measures broadband frequency range spectrum and is commonly used in near 


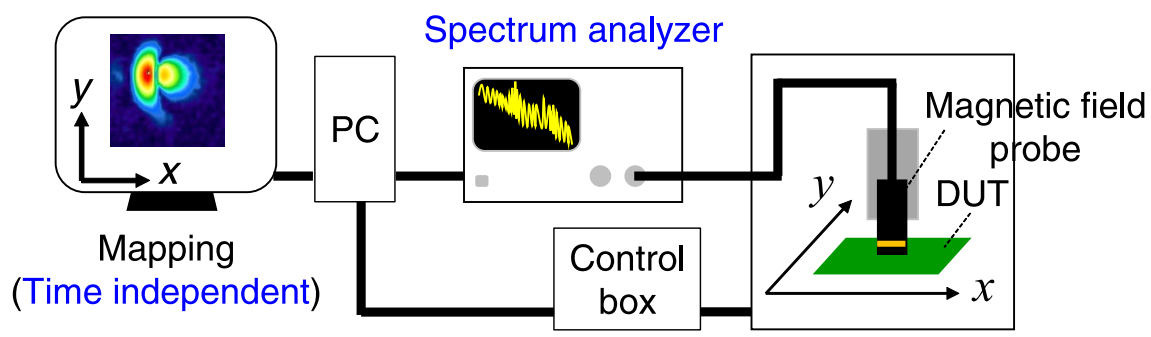

(a) Conventional configuration for evaluating static near magnetic field

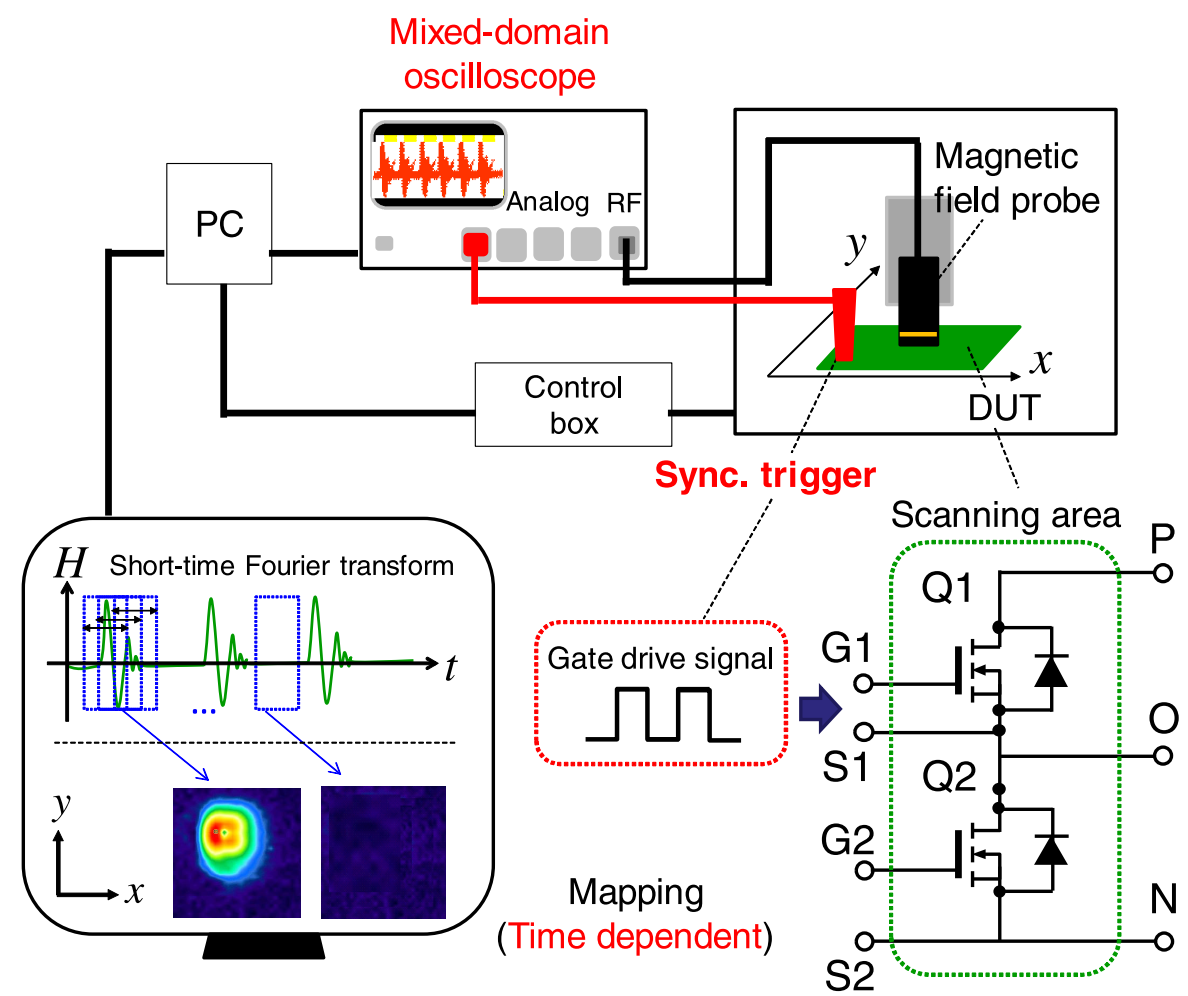

(b) Proposed configuration for evaluating dynamic near magnetic field

Fig. 1. System configuration of near magnetic field scanning.

magnetic field measurement systems. However, time variations of the magnetic field are not attainable for the conventional spectrum analyzer. The EMI noise source in a switching power converter is transient voltage and current variation caused by the switching operations of power semiconductor devices. Therefore, visualization of the time-related noise component behavior is useful to clarify the EMI noise generation mechanisms in power electronics circuits. This study focuses on the dynamic noise current distribution in a power module along with the switching operation.

Fig. 1(b) depicts the developed system configuration for dynamic near magnetic field measurement. The proposed system uses a mixed-domain oscilloscope (MDO) instead of a spectrum analyzer. A magnetic field probe is connected to the $\mathrm{RF}$ input of the real-time spectrum analyzer channel of MDO. The gate signal of the MOSFET is measured with an analog channel and is used as the synchronous trigger for the real-time spectrum analyzer. Time synchronization of measurement is secured for the periodic steady state operation. The PC controls the magnetic probe position and records the acquired data of MDO. The stored data of time- 

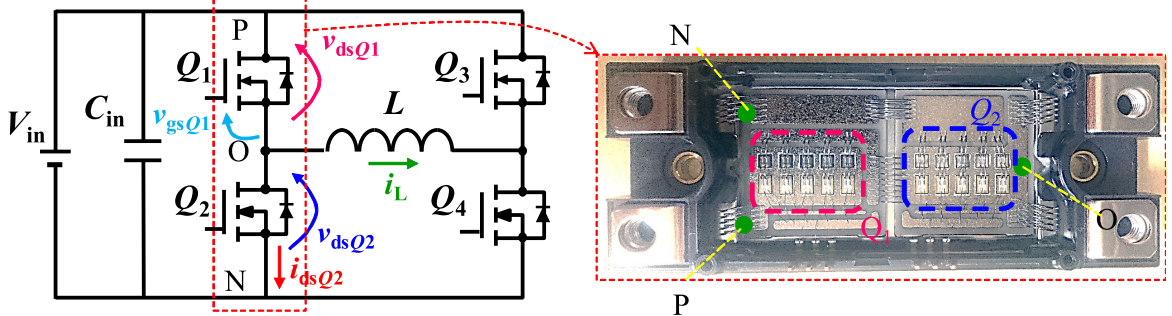

(a) Test circuit configuration and device under test of the $\mathrm{SiC}$ power module
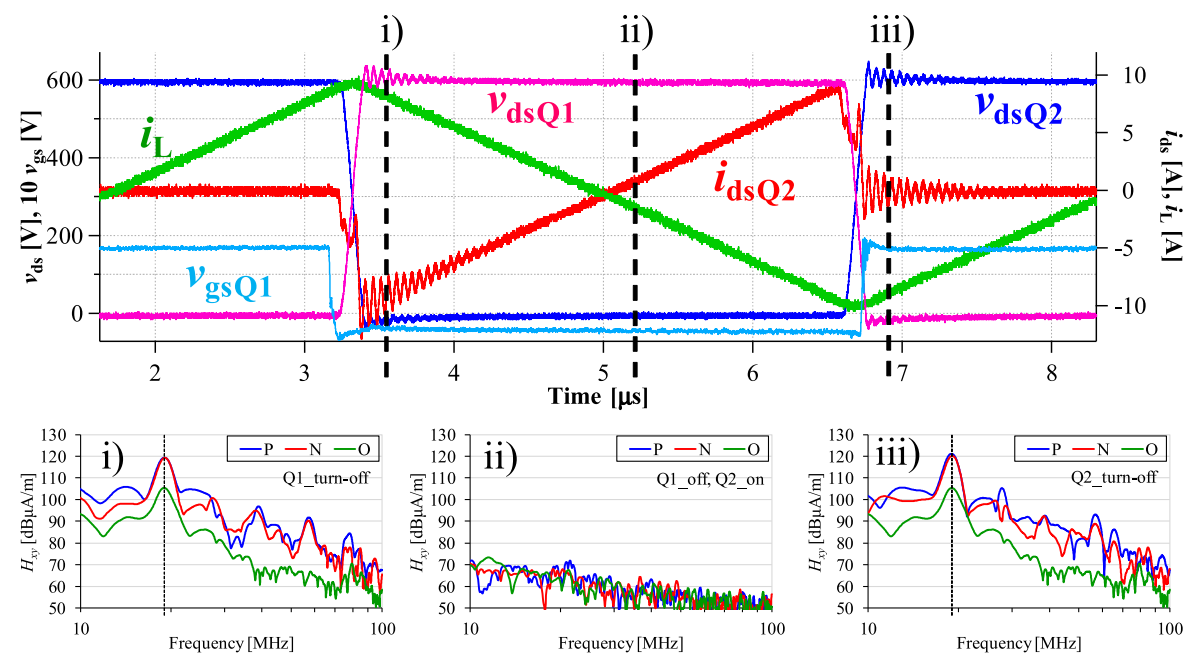

(b) Time domain waveform of circuit voltage and current and frequency spectrum of near magnetic field at $\mathrm{P}, \mathrm{N}, \mathrm{O}$ terminals

Fig. 2. Characterization of time-synchronized near magnetic field spectrum in the $\mathrm{SiC}$ power module.

synchronized near-field intensity for the respective measurement point are analyzed via the short-time Fourier transform (STFT) in the time-frequency mixed domain [10] as a function of the real-time spectrum analyzer. The developed system measures the time evolution of frequency spectrum of near magnetic field along with the triggered switching operation and scans spatially in $2 \mathrm{D}$. The measured time-synchronized near magnetic field intensity for scanned power module is presented as a colored graphic map for the specific time and frequency. A sequence of the magnetic field intensity map, shown as a movie, visualizes the dynamic characteristics of noise current propagation along with the switching operation. An experimental example of this approach is shown in the following section.

\section{Experimental results of dynamic noise current distribution and propagation in the SiC power module}

Fig. 2(a, left) shows the test circuit diagram for identifying the noise current distribution in the power module. DUT is a commercially available SiC half-bridge power module (ROHM, BSM120D12P2C005), as demonstrated in Fig. 2(a, right). There are five parallel-connected MOSFETs with five anti-parallel SiC schottky barrier diodes in each arm. The test circuit constitutes a single-phase DC-AC fullbridge inverter. The power supply and smoothing capacitor are connected to the $\mathrm{DC}$ bus, and the load inductor is connected to the AC bus. The input DC voltage is 
$V_{\text {in }}=600 \mathrm{~V}$, and $100 \mu \mathrm{H}$ inductive load current is $i_{L}= \pm 10 \mathrm{~A}$. The switching frequency is set to $148.5 \mathrm{kHz}$ and duty ratio $D=0.465$. The complementary switching operations of MOSFETs $Q_{1}$ and $Q_{2}$, as well as $Q_{3}$ and $Q_{4}$ periodically repeat charge and discharge energy to the load inductor, and real power consumption is negligible for this circuit.

A mixed-domain oscilloscope (Tektronix, MDO4104B-6) is triggered with the $v_{g s Q 1}$, and the RF signal is simultaneously captured. Then, time-frequency spectrum (spectrogram) of near magnetic field is obtained with synchronizing switching operation of $Q_{1}$. The measured timing of near magnetic field is shown in Fig. 2(b). The magnetic field probe (NEC Engineering, MP-10L) measures the flux generated by the currents flowing high-side MOSFET $Q_{1}$ (near the P terminal), low-side MOSFET $Q_{2}$ (near the $\mathrm{N}$ terminal), and the load inductor (near the $\mathrm{O}$ terminal). Fig. 2(b) i)-iii) show the time-synchronized frequency spectrum of the near magnetic field in $10-100 \mathrm{MHz}$ calculated via STFT, which correspond to the time i)-iii) in the measured time-domain voltage and current of the tested circuit. There is no significant spectrum peak in $10-100 \mathrm{MHz}$ when the MOSFET is conducting the load current in state ii), as illustrated in Fig. 2(b). The near magnetic field exhibits the highest spectrum peak at $19-\mathrm{MHz}$ associated with the switching operations (states i) and iii)). This corresponds to the ringing oscillation frequency of voltage and current induced by the switching operation. The oscillation results from the resonance between the output capacitance of the transistor in blocking condition and parasitic inductance in the power loop. The power loop is the current path from the input DC-link smoothing capacitor $C_{i n}$ through the half-bridge leg, $Q_{1}$ and $Q_{2}$, and back to the input capacitor. The output capacitance $C_{o s s}$ of $Q_{2}$ is $850 \mathrm{pF}$ at $600 \mathrm{~V}$ DC bus voltage, which is measured by a curve tracer (Agilent, B1505A). The parasitic inductance of power loop Lloop is identified as $82.5 \mathrm{nH}$, which is estimated from the ringing oscillation frequency $f(19-\mathrm{MHz})$ and the output capacitance $C_{\text {oss }}(850 \mathrm{pF})$ as $L_{\text {loop }}=\frac{1}{\left(4 \pi^{2} f^{2} C_{\text {oss }}\right)}$. The 19-MHz spectrum peak of near magnetic field at $\mathrm{O}$ terminal is $15 \mathrm{~dB}$ smaller than that at $\mathrm{P}$ and $\mathrm{N}$ terminals. The results suggest that the noise current, resulting from the switching operations, predominantly flows through the power loop. It does not smear the load inductor since the output loop has large impedance in high frequency.

Fig. 3 demonstrates an example of extracted 1-MHz component conducting current distribution in the power module with the scanned time-synchronized near magnetic field measurement. The extracted $1-\mathrm{MHz}$ component appears as the seventh order harmonic of switching frequency. The difference in harmonic current distribution with time is clearly attributed to circuit operating conditions. The developed time-synchronized measurement of scanning system can extract the current distribution in a power module at the specific time and frequency.

Fig. 4 illustrates the $19-\mathrm{MHz}$ noise current component propagation in the power module for $Q_{2}$ turn-off operation. The intensity of time-synchronized 19$\mathrm{MHz}$ near magnetic field in the power module is represented as a colored graphic animation. A sequence of the colored maps with 40-ns step represents the noise current propagation. The load inductor current forces to turn on the anti-parallel diodes of $Q_{1}$ for the turn-off operation of $Q_{2}$. After the $Q_{1}$ turn-on operation, the charging current flows from the DC input capacitor to the output capacitance of $Q_{2}$ 


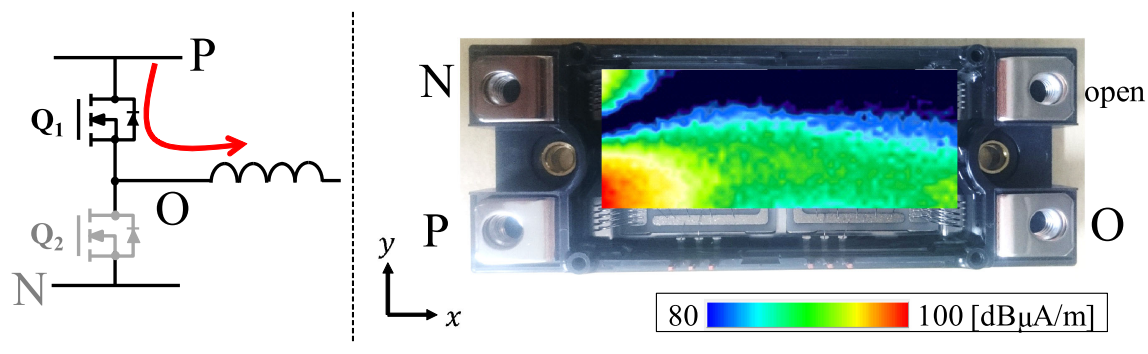

(a) $Q_{1}$ conducting, $Q_{2}$ non-conducting

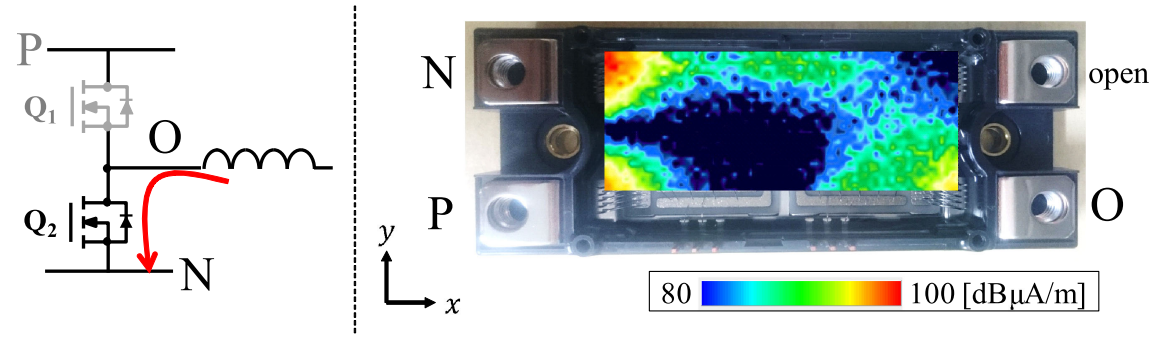

(b) $Q_{1}$ non-conducting, $Q_{2}$ conducting

Fig. 3. Measured $1 \mathrm{MHz}$ near magnetic field distribution for conduction current evaluation.
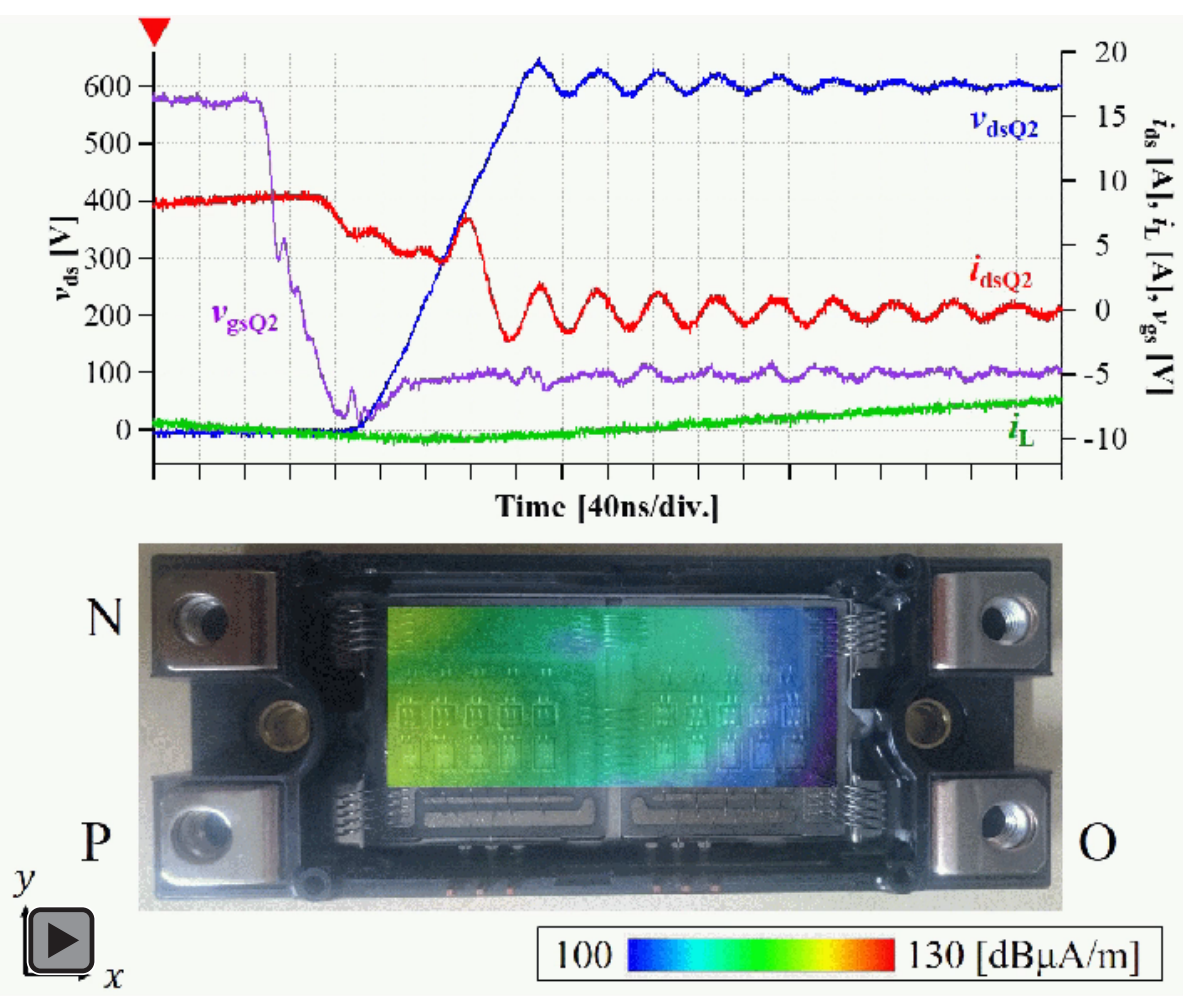

Fig. 4. Dynamic characteristics of noise current propagation (19 MHz) in $Q_{2}$ turn off operation. Movie file attached. Movie can be activated by clicking on the icon.

via the parasitic inductance in the power loop. This causes 19-MHz LC resonance current in the power loop of the tested circuit. The noise current behavior shown in Fig. 4 suggests that the $19-\mathrm{MHz}$ noise current propagates from $\mathrm{P}$ terminal to $Q_{1}$ and $Q_{2}$. The 19-MHz noise current component does not smear out on the load inductor current. This is in agreement with the result presented in Fig. 2(b). Thus, the 
developed time-synchronized near magnetic field measurement can visualize the dynamic noise current behavior associated with the circuit operations. Further studies are required to evaluate EMI noise characteristics of the circuit considering the visualized noise current propagation to design a low-EMI power module and power conversion circuit.

\section{Conclusion}

Herein, we discussed the dynamic behavior of the noise current distribution in a power module based on scanned time-synchronized near magnetic field measurement. The proposed approach can evaluate the noise current distribution associated with switching operations, offering a new perspective that cannot be achieved via the conventional frequency domain analysis. The experimental results shown in this report ensure the validity of the time-synchronized near magnetic field scanning system to identify the EMI noise source and propagation of the power converter. It is useful to estimate the dynamic noise current distribution in the early stage of designing wiring layout and packaging for the next generation power modules with low-EMI characteristics, which is a point for discussion in our future study.

\section{Acknowledgments}

This work was partially supported by Council for Science, Technology and Innovation (CSTI), Cross-ministerial Strategic Innovation Promotion Program (SIP), "Next-generation power electronics" (funding agency: NEDO). 